Kompass

Pneumologie

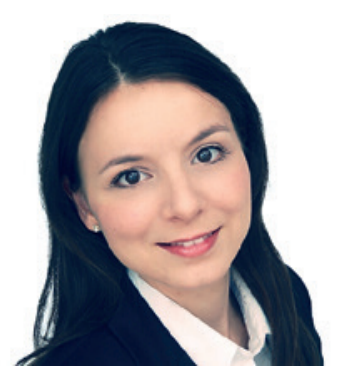

\title{
Eosinopenie als Biomarker des Schweregrads einer COVID-19-Infektion
}

Panagiota Xanthoulia, b

a Sektion Pulmonale Hypertonie, Thoraxklinik am Universitätsklinikum Heidelberg, Heidelberg, Deutschland; b Rheumatologische Ambulanz, Zentrum für Innere Medizin V - Klinik für Hämatologie, Onkologie und Rheumatologie, Heidelberg, Deutschland

Abstract aus Valverde-Monge M, Cañas JA, Barroso B, et al.: Eosinophils and chronic respiratory diseases in hospitalized COVID-19 patients. Front Immunol. 2021;12:668074.

\section{Keywords}

COPD (chronic obstructive pulmonary disease) · COVID-19 · OSA (obstructive sleep-apnea) · Asthma · Chronic respiratory diseases · Eosinopenia · Eosinophils

\section{Abstract \\ Background: Studies on the role of eosinophils in coronavirus disease 2019 (COVID-19) are scarce, though available findings sug- gest a possible association with disease severity. Our study ana- lyzes the relationship between eosinophils and COVID-19, with a focus on disease severity and patients with underlying chronic respiratory diseases.}

Methods: We performed a retrospective analysis of 3018 subjects attended at two public hospitals in Madrid (Spain) with PCRconfirmed SARS-CoV-2 infection from January 31 to April 17, 2020. Patients with eosinophil counts less than $0.02 \times 10^{9} / \mathrm{L}$ were considered to have eosinopenia. Individuals with chronic respiratory diseases $(n=384)$ were classified according to their particular under- lying condition, i.e., asthma, chronic pulmonary obstructive disease, or obstructive sleep apnea.

Results: Of the 3018 patients enrolled, 479 were excluded because of lack of information at the time of admission. Of 2539 subjects assessed, 1396 patients presented an eosinophil count performed on admission, revealing eosinopenia in 376 cases (26.93\%). Eosinopenia on admission was associated with a higher risk of intensive care unit (ICU) or respiratory intensive care unit (RICU) admission (OR:2.21; 95\%Cl:1.42-3.45; $p<0.001$ ) but no increased risk of mortality ( $p>0.05)$.

Conclusion: Eosinopenia on admission conferred a higher risk of severe disease (requiring ICU/RICU care), but was not associated with increased mortality. In patients with chronic respiratory diseases who develop COVID-19, age seems to be the main risk factor for progression to severe disease or death.

() 2021 Valverde-Monge, Cañas, Barroso, Betancor, Ortega-Martin, Gómez-López, Rodríguez-Nieto, Mahíllo-Fernández, Sastre and Del Pozo 


\section{Transfer in die Praxis}

\section{Hintergrund}

Biomarker für die Früherkennung des Schwergrads einer COVID19-Infektion sind mangelhaft [1]. Die Bestimmung der Akut-PhaseProteine (C-reaktives Protein (CRP), D-Dimere, Ferritin, Procalcitonin u.a.) ist teuer, zeitaufwendig und häufig ungenau [1], was das Gesundheitssystem zunehmend finanziell belastet. Die Bestimmung von Eosinophilen und der Nachweis und die Persistenz der Eosinopenie (Mangel von Eosinophilen) deuten einen komplizierten Verlauf [2] der COVID-19-Infektion an [3]. Weitere Studien über die Funktion der Eosinophilen während der COVID-19-Infektion sind erforderlich.

\section{Ergebnisse der Studie}

In dieser Studie aus Madrid haben Valverde-Monge et al. die Bedeutung der Eosinophilenzahl (Eos) bei Patienten mit PCR-gesicherter neu diagnostizierter COVID-19-Infektion sowohl während der Erstdiagnose als auch im Verlauf geprüft. Insgesamt wurden 3018 Patienten vom 31.01.2020 bis zum 17.04.2020 untersucht. Eosinopenie wurde definiert als absolute Eos $<0,02 \times 10^{9} / \mathrm{L}$. Eingeschlossen waren 2539 Patienten. Insgesamt sind 320 Patienten verstorben, 86 davon hatten Lungenerkrankungen ( $p<0.0001$ ). Bei 1396 Patienten der gesamten Kohorte erfolgte die Bestimmung von Eos bei der Aufnahme und bei 627 bei Aufnahme und Entlassung. Insgesamt 26,93\% (376/1396) hatten Eosinopenie. Erhöhte D-Dimere und Lymphopenie waren die Laborwerte in dieser Kohorte, die mit signifikant erhöhter Mortalität assoziiert waren. Das Alter war der Hauptrisikofaktor für höhere Mortalität in dieser Kohorte. Es zeigte sich eine signifikante und kontinuierliche Abnahme der Eos im Vergleich vor Infektion vs. bei COVID-19-Diagnose vs. bei Entlassung bei den Patienten mit Lungenkrankheit unabhängig von der Art der Erkrankung (Asthma etc.). Die Abnahme der Eos bei Aufnahme vs. bei Entlassung war signifikant sowohl in der Gruppe der Lungenkranken $(p<0.0001)$ als auch in der Gruppe der nicht Lungenkranken $(p<0.001)$. Die Eosinopenie war mit signifikant erhöhtem Risiko einer Aufnahme auf eine Intensivstation $(p<0001)$ in beiden Gruppen, aber nicht mit Mortalität assoziiert.

\section{Fazit für die Praxis}

Das Screening von COVID-19-infizierten Patienten sollte u.a. auch die Bestimmung von Eosinophilen beinhalten, da eine niedrige Eosinophilenzahl mit hoher Hospitalisationsrate assoziiert ist.

\section{Disclosure Statement}

Hiermit erkläre ich, dass keine Interessenskonflikte in Bezug auf den vorliegenden Kommentar bestehen.

\section{Zweitveröffentlichung}

Dieser Beitrag wurde erstveröffentlicht in Kompass Autoimmun 2021;3:176-177.

\section{Literatur}

1 Gao Y-D, Ding M, Dong X, et al.: Risk factors for severe and critically ill COVID-19 patients: A review. Allergy. 2021;76(2):428-455.

2 Gil H, Bouldoires B, Bailly B, et al.: Eosinopenia in 2018. Rev Med Interne. 2019:40(3):173-177

3 Lindsley AW, Schwartz JT, Rothenberg ME: Eosinophil responses during COVID-19 Infections and coronavirus vaccination. J Allergy Clin Immunol. 2020;146(1):1-7.

Korrespondenz an:

Dr. Panagiota Xanthouli, panagiota.xanthouli@med.uni-heidelberg.de 